\title{
Endogenous Ketamine-Like Compounds and the NDE: If So, So What?
}

\author{
Rick J. Strassman, M.D. \\ Victoria, British Columbia
}

ABSTRACT. This commentary on Karl Jansen's ketamine model for the neardeath experience expands upon and raises additional questions about several issues and hypotheses: self-experimentation as a source of data; ketamine's similarities to and differences from classical hallucinogens; the need for quantification of unusual subjective states; clinical research and toxicological implications of this model; drugs as gateways to "religious" states; and "evolutionary" versus "religious" significance of naturally occurring compounds released in the near-death state. I suggest future research that could help explicate several of these areas.

Karl Jansen's paper cogently summarized the pre-clinical pharmacology of glutamate and N-methyl-D-aspartate (NMDA) receptors relative to the effects of ketamine, a dissociative anesthetic with "psychedelic" properties at subanesthetic doses. He also reviewed the less voluminous human literature on subjective effects of ketamine. He suggested that since ketamine, a "neuroprotective" NMDA receptor antagonist, elicits an alteration of consciousness with features similar to those described by individuals having a near-death experience (NDE), that an endogenous ketamine-like substance is released when an organism's brain is faced with potentially life-threatening circumstances. The neuroprotective effects of such an endogenous NMDA antagonist thus are called upon by the organism to reduce the threat of brain damage. The NDE results from the psychoactive properties

Rick J. Strassman, M.D., was formerly Associate Professor of Psychiatry at the University of New Mexico School of Medicine. Reprint requests should be addressed to Dr. Strassman at 1783 Rockland Avenue, Victoria, British Columbia, Canada V8S $1 \mathrm{X} 1$. 
of these ketamine-like neuroprotective agents. Indeed, as is noted, "endopsychosins," with receptor binding profiles similar to ketamine, have been found in rodents.

The breadth of data and hypotheses presented by Jansen provides a springboard for discussing a wide variety of complex and intriguing issues. These include:

1) self-experimentation as a source of data;

2) ketamine versus "classical" hallucinogens, with respect to a cogent comparison of their subjective effects and the nature of their symptomatic overlap with the NDE, and with respect to their suitability for candidacy as an endogenous NDE-causing agent, or "NDE-ogen";

3) quantification of highly unusual mental states, including those elicited by NDEs and drugs;

4) clinical research and toxicology implications of the model;

5) the use of drugs as gateways to "religious" states; and

6) evolutionary/scientific or religious/vitalistic significance of endogenous NDE-ogens.

\section{Self-Experimentation as a Source of Data}

Jansen referred to the literature on human subjective responses to ketamine. He also drew upon his own NDEs and experiences with ketamine. He also suggested that his own experiences with the classical hallucinogens, that is, N,N-dimethyltryptamine (DMT) and lysergic acid diethylamide (LSD), demonstrate a lack of similarity between the effects of ketamine and these latter drugs.

Self-experimentation is a valuable and long-standing tradition in medical research, particularly with psychoactive materials (Szara, 1957). In this case, a discussion of the circumstances under which such experiences took place would strengthen Jansen's hypothesis. For example, why were these drugs administered, what doses were administered and by whom, and how many administrations? Were these double-blind studies? Was there an active placebo control for ketamine, especially one with a similar time course and dissociative properties (for example, nitrous oxide, carbon dioxide, short-acting parenterally-active tryptamines)? These all are important variables helping one assess the validity of experimental claims regarding psychoactive drug effects. 
In the case of Jansen's NDEs, a fuller description also would shed light on the cogency of his comparisons of ketamine with the NDE. What happened to him? How close was he to death? What was the nature of the resuscitative interventions, if any? What did he see, think, and feel?

\section{Ketamine Versus “Classical” Hallucinogens}

\section{How Well Does Ketamine Replicate the NDE Relative to Classical Compounds?}

Jansen's conclusion that ketamine "can reproduce all the features of the NDE," while classical hallucinogens do not, was based upon comparing subjective effects of NDEs, ketamine, and classical compounds. This is an extraordinarily important point, and one that requires continued elaboration.

"Classical" hallucinogens include LSD, psilocybin, mescaline, and DMT. These drugs have been administered to thousands of patients and normal controls in clinical research, both psychopharmacologic and psychotherapeutic, over the last 50 years (mescaline for over 100 years).

While Jansen referred to previously published papers describing psychological effects of both ketamine and the NDE, his assertion that ketamine and NDEs are identical would have been strengthened by a more rigorous comparison, particularly regarding the nature of any differences between the two. Attention to differences might suggest gaps in theory, gaps that could be filled by additional hypothesis-driven experimentally-derived data.

Regarding differences, I have been struck by the fearful experiences many anesthetized patients describe as they are awakening from ketamine anesthesia, when blood levels compare to those attained with nonmedical or "recreational" use. These so-called "emergence phenomena," while partaking of some of the particular perceptual and cognitive properties of the NDE, often are not pleasant nor desired to be repeated, and lack the equanimity and reinforcing effects that recreational ketamine users describe. Nor are they felt to be beneficial over the long run, that is, lessening fear of death and enhancing appreciation of life.

In addition, the highly addictive nature of the ketamine experience (for example, John Lilly's injecting himself with ketamine every hour 
for weeks on end) does not comport with how one describes the consequences of the NDE. I am not aware of NDErs compulsively repeating the near-death state, for example, by frequent suicide attempts. Perhaps they become more risk-taking after an NDE, but this does not necessarily imply a desire to repeat the NDE as much as a lack of fear of death. On the other hand, it may be that increased risk-taking is an antecedent in those who compulsively use ketamine and other psychoactive drugs.

The lack of NDE-like properties of the classical psychedelics is not established. In fact, the research team at the Spring Grove State Hospital in Maryland used classical compounds such as LSD, psilocybin, and N,N-dipropyltryptamine (DPT) in their work with the terminally ill. This was because the overlap between descriptions of their volunteers' "peak" psychedelic experiences, mystical states, and NDEs seemed so pronounced (Pahnke, Kurland, Unger, Savage, and Grof, 1970).

Whenever effects of psychoactive drugs are described, one must pay careful attention to "set" and "setting," that is, expectations of the volunteer, and interpersonal and physical nature of the drug-taking environment. Along these lines, the intensification and distortion of environmental stimuli Jansen referred to as a hallmark of LSD effects, for example, could be powerfully altered simply by closing the eyes and lying still. Dissociation and dreamlike imagery, as described with ketamine use, very quickly develop in these circumstances.

Also important to consider is the effect of rapidity of onset of drug effects influencing interpretation of those effects. For example, the NDE-like nature of ketamine effects may have much to do with the rapidity of onset seen with intramuscular or intravenous administration. The absence of much time to prepare for (and defend against) effects, and the dissociative nature of these effects, may combine to be interpreted as death, or a deathlike state, since the sense of self is so heavily invested in the integrity of the body's constantly supplied perceptual input.

The classical hallucinogen with a similar temporal profile of activity is DMT. In our DMT studies at the University of New Mexico, the onset of intravenous DMT effects is nearly instantaneous, peak effects culminate at $1 \frac{1}{2}$ to 3 minutes, and are resolved by 20 to 30 minutes (Strassman, Qualls, Uhlenhuth, and Kellner, 1994). Several volunteers, none of whom had had a spontaneous NDE in the past, were convinced they had died as a result of the DMT injection, and 
some even hallucinated resuscitation efforts being applied to their near lifeless bodies. Others viewed themselves lying in the hospital bed. Many said that they no longer feared death after having undergone a high dose of intravenous DMT. Many volunteers also met and interacted with "entities," "angels," "beings of light," and "guides," and "knew God" as a result of drug administration in our research setting. One volunteer, in response to an observing students' comparing one of her seemingly bizarre dreams to the volunteer's description of his just-completed DMT session, said, "What you are describing is a dream. This was different; this was real." One volunteer even said, "People would all kill themselves if they knew how wonderful this place beyond everyday life is."

Finally, I do not know of habitual repetitive use of DMT, as can occur with ketamine, despite the highly pleasurable effects of DMT. This is not due to tolerance developing to closely spaced DMT administrations, as we have demonstrated this does not occur (Strassman, Qualls, and Berg, in press).

\section{Are There Classical Hallucinogen Candidates for an Endogenous NDE-ogen?}

Jansen argued persuasively for a ketamine-like NMDA receptor antagonist as a naturally occurring NDE-ogen. However, it may be that other psychoactive compounds similarly affect brain function during dire physiological circumstances.

DMT, for example, is a naturally occurring hallucinogenic tryptamine, found in human blood, urine, and spinal fluid in many studies. Enzymes and precursors necessary for its production have been found in several species, including humans (Gillin, Kaplan, Stillman, and Wyatt, 1976). The "endopsychosins" with ketamine-like binding properties, on the other hand, have only been found in rodents, and there are few data concerning their existence since the initial 1984 paper describing them.

In summary, the subjective responses to, and longer-term effects of, the emergence phenomenon, recreational ketamine use, and NDEs, as well as ketamine addicts' loss of control and consistently euphoric responses to the drug, are important data to consider when proposing a phenomenological identity of NDEs with ketamine inebriation. In addition, a broader interpretation of the classical hallucinogen human literature (Strassman, 1995), and consideration of 
endogenous tryptamine psychedelics as naturally-occurring NDEogens, suggest that classical compounds provide a viable alternative or complement to a ketamine/NMDA-antagonist theory of NDEs.

\section{Quantifying Unusual Mental States}

As noted in the above discussion, there is a need for more finely tuned methods of comparing the nature of the subjective effects of ketamine, classical psychedelics, and the NDE.

Comparison of subjective effects of drug-induced and nondrug-induced altered states of consciousness requires both careful clinical observation and interviews, in addition to some quantitative scoring of phenomena. Previous scales of subjective drug effects, such as the older LSD scales, predicated their questions and scoring methods on the assumption that psychedelics produced pathological states of mind-at least highly regressed, if not frankly psychotic. The standard scale, the Addiction Research Center Inventory (ARCI) compares novel drugs' effects to norms established for reference drugs, such as LSD, morphine, and benzedrine (Haertzen and Hickey, 1987). In generating normative data, the most popular LSD scales (Abramson, Jarvik, Kaufman, Kornetsky, Levine, and Wagner, 1955; Linton and Langs, 1962) and the ARCI used psychedelic-naive volunteers, who were told little if anything about what drug effects might be. In the case of the ARCI, abstinent narcotic addicts serving time in a penitentiary provided these data.

To remedy the problems both of assuming a priori the pathological nature of psychedelic effects, and of naive and/or psychopathic volunteers, we developed a new rating scale, the Hallucinogen Rating Scale (HRS), which may have relevance to the issues under discussion. The HRS was developed by interviewing healthy well-functioning hallucinogen users who found these drugs highly pleasurable and interesting, and the HRS was modified during our early work with intravenous DMT. While the development and initial data from the HRS are beyond the scope of this commentary (Strassman, Qualls, Uhlenhuth, and Kellner, 1994), how we clinically clustered questions is relevant.

We grouped the $120+$ questions of the HRS by "clinical clusters," such as "perception," "cognition," "somatic effects," "volition," and "affect." These are "mental status examination" categories by which a clinical psychiatric examination takes place in any setting. This man- 
ner of clustering items provided as good resolution of subtle dose effects as a purely statistical (principal components) analysis, and was superior to multiple biological variables in resolving dose effects.

While the HRS is still in development, we do have preliminary data from an ongoing ketamine study in the United States suggesting that subanesthetic "psychedelic" doses of ketamine are remarkably similar to those of hallucinogenic doses of DMT. In addition, there are other preliminary data from a Canadian study showing that amphetamine does not produce the same type of psychedelic profile seen with DMT and ketamine.

\section{Clinical Research and Toxicology Implications}

It is often implicitly suggested, if not overtly stated, that NDEs are somehow good for you. The bliss, guidance, and reassurance by otherworldly beings, and grace-like features associated with the actual NDE; the beneficial changes in lifestyle or personal philosophy; improvement of mental health or substance abuse problems; and decreased fear of death all add up to sounding like a beneficial experience. The outcome sounds similar to that of a religious conversion, or a mystical state. Similar claims are well known from proponents of high dose hallucinogenic drug "psychedelic" psychotherapy.

While the model put forth by Jansen was primarily explanatory, it also might be used to support the use of ketamine to induce NDEs or NDE-like phenomena. These human studies could further elucidate the effects and mechanisms of action of ketamine, and explicate crucial similarities and differences among the subjective states induced by ketamine, classical hallucinogens, and NDEs.

Jansen's suggestion that ketamine be given to those who have had a spontaneous NDE and a careful comparison made could be a pivotal study, and one that would benefit from the quantification of subjective states possible with the HRS. As well, DMT and/or other short-acting dissociative mind-altering agents, such as nitrous oxide and carbon dioxide, could be compared in the same volunteers. A simpler, useful, but less rigorous study, lacking the within-subjects design of the aforementioned one, would administer the HRS to those who have had a spontaneous NDE, and compare scores for their NDE with previously established norms for ketamine and other agents obtained from an independent sample. 
Taken to its logical ends, this psychopharmacological approach may lead to experiments in which brains of immediately deceased individuals were assayed for endogenous NDE-ogens. The psychopharmacologists among us would want to blockade the effect of this NDE-ogen using selective antagonists, and then compare responses to nonpretreated immediately deceased! The ethical and technical issues boggle the mind.

More likely, however, is that studies could be undertaken with a therapeutic intent, where people in distress are treated with a ketamine-induced altered state. Such conditions might include drug abuse, posttraumatic stress disorder (PTSD), and the pain and suffering associated with terminal illness, ones often included in discussions of psychedelic-assisted psychotherapy. These are often intractable problems, frequently frustrating caregivers and family. There is great appeal to the concept of a magic bullet, one or several peak experiences such as an NDE or mystical/religious state that break through resistances and intractability, give a glimmer of hope and faith, and the conviction of a "higher reality." Having glimpsed this new view, patients can now use it as a compass and reference point hereafter. Or, more specifically with regard to the terminally ill, if NDEs reduce fear of death, and ketamine produces an NDE, then perhaps a "dry run" using ketamine could ease this fear, and indeed, ease their real death.

The exclusively biologically-based clinician might at some point be able to point to clinical or preclinical data suggesting that these conditions-drug abuse, PTSD, fear of death-result from "excess glutaminergic tone." The logical treatment would be an NMDA antagonist, such as ketamine. If the psychedelic effects were seen as an adverse side effect, agents that were effective antagonists and free of psychological effects might be developed, or the psychological effects selectively blockaded.

Before such therapeutic studies are undertaken, however, I believe that several issues should be thoroughly appreciated and inform any discussion preceding human therapeutic trials. The first three issues are common to well-conceived, hypothesis-driven clinical trials of new therapeutic agents; the fourth ventures into the murkier realms of pharmacological gateways to religious states.

The first issue concerns the basis for using ketamine as a therapeutic agent; that is, does it indeed reproduce an NDE? If careful human work demonstrates that it does, then a cogent rationale for applying an NDE to a clinical condition must be presented. If 
ketamine does not reproduce an NDE, then its use as a treatment modality should not rely upon its similarities to an NDE. This is not to say there may not be psychotherapeutic uses of ketamine and other more typical hallucinogens, but that the bases for using them as experimental therapeutics must be clearly articulated.

A second issue is that of the risk:benefit ratio. If acute and longerlasting adverse psychological reactions to ketamine occur, as they do with classical psychedelics (Strassman, 1984), it would be important to plan for this contingency. This is particularly germane if terminally ill patients were studied, and little time remained to remedy the consequences of a frightening ketamine-induced near-death state.

Third, ketamine has been reported to be neurotoxic in certain animal models (Olney, Labruyere, and Price, 1989), its toxicity reversed by diazepam (Olney, Labruyere, Wang, Wozniak, Price, and Sesma, 1991). Since diazepam also prevents the "psychotomimetic" emergence phenomena seen in humans, it is possible that the NDE-like symptoms of ketamine are secondary to neurotoxicity. This also suggests that development of ketamine-analogues with psychedelic effects but lacking neurotoxicity will be difficult.

\section{Drugs as Gateways to Religious States}

The final issue I would like to raise is that of "set" and "setting" for the use of ketamine. This begins to touch upon the more troubling and controversial relationship between drugs and religious experience, a topic that continues to stir rancorous debate. There is even now interest in renaming these drugs "entheogens," generating the divine/God within. In my own case, religious claims for these drugs contributed to my interest in studying these drugs in a clinical research setting. Part of this debate addresses the question whether hallucinogens, including ketamine, are inherently "good" drugs for people to take because they provide actual paths to personal growth, either religious or psychological.

These issues should be appreciated in designing practical elements of hallucinogen administration to humans, particularly therapeutic trials. Who should be given ketamine, and how? Who should give ketamine, and how? Should administering clinicians have their own experience with the drug? Do they need a background in religion and philosophy to hold empathically, support, and nourish the experience of their patients? Do the conditions elicited by ketamine and 
other psychedelics properly belong within the purview of psychiatric clinicians and research scientists?

What do we do with the "religious" utterances of our patients? For if these drugs do elicit states best described in religious and spiritual terms, does the administering clinician essentially dash these beliefs by understanding and interpreting them as purely biochemical perturbations with subjective counterparts, never mind intimations of immortality and divine revelation, no matter how powerfully held by the experiencer these latter beliefs are? Is there something pharmacologically useful in the chemical nature of these drugs, that by their revelatory properties could substitute for or enhance years of long self-analysis and discipline? Should we tell patients that?

Our research team was alert to these factors while working with DMT. While our studies were not explicitly therapeutic in intent, part of my interest in performing this research was to assess the impact of a "neutral" set and setting on a psychedelic drug-induced extreme alteration in consciousness. I wanted to see if DMT was "good," "bad," or "indifferent" in its "nature."

My conclusion after administering more than 400 doses of DMT to over 50 relatively healthy, experienced hallucinogen-using volunteers, is that there is nothing inherent about the drugs that has a beneficial effect. Neither are they pharmacologically in and of themselves dangerous. The nature of, and sequelae to, the experience are determined by a complex interplay of the drug's pharmacology, the nature (states and traits) of the volunteer at the time of drug administration, and the nature of the relationship between the individual and his or her physical and psychological environment.

The volunteers who benefitted most from their DMT sessions were those who probably would have gotten the most out of any "trip," drug or otherwise. Those who benefitted least were those who were the most novelty-sated and less likely to incorporate new concepts, perceptions, or feelings, the novelty being more important than what was novel. And the most difficult sessions took place in various degrees of contributions of two factors. These were, first, an inability voluntarily to give up the internal dialogue and body-awareness; and second, ambiguous relationships between the volunteer and those in the room at the time (usually guests or students introduced to the volunteer right before drug administration). Thus, the "religious," "adverse," or "banal" effects resulting from drug use depended more upon the volunteers and what they and those in the room brought 
to the session, than from any indwelling characteristic of the substance itself.

For example, two people we studied had minimal responses to a high dose of intravenous DMT (I do not know if there are cases of lack of response to ketamine, too). One of these volunteers was a highly experienced meditator, while the other was a bartender who preferred sailing. The first volunteer had a meditation-induced religious experience some years before, and my facile explanation was that his "enlightenment" experience might have changed his neurophysiology in such a way as to raise his threshold radically for druginduced mystical states. However, I am more at a loss explaining the lack of response in the other volunteer. We joked with him that he was already enlightened but just didn't know it!

There are clinical research implications of these issues, which will affect experimental designs and the nature of the outcome data. If acute drug-induced alterations in consciousness are to be used in a traditional clinical research setting, and therapeutic intent is explicit, I believe the issue is not whether or not a psychedelic drug by itself is useful: it is not. Neither is the issue whether or not "NDEs," druginduced or spontaneous, are therapeutic. The issue is rather whether a drug affects psychological processes, and if so, how such effects can be turned to enhance the therapeutic process. And, if these subjective effects are best conceptualized in "religious" terms, is it necessary to incorporate pastoral counseling and/or 12-Step or other "spiritual" modalities and explanatory models into the design of any study?

In summary, therefore, an appropriate research model might treat patients in an accepted manner, and compare results when a psychedelic drug or appropriate placebo is added. The interaction of drug, psychology, biology, and social environment could all be assessed carefully. This design would clarify the nature of the drug interactions with the particular clinical condition and its treatment in a particular setting. For example, is there a relationship between ketamine's effects on motivation or insight, and abstinence from cocaine use or frequency of flashbacks in PTSD?

\section{Scientific/Evolutionary or Religious/Vitalistic Significance of Endogenous NDE-ogens}

This is the most speculative, but among the most compelling, area raised by any biological model of the NDE. That is, "If so, so what?" 
Why would nature design biology in this way? Is it a coincidence, or is it "for a reason"?

Jansen did not include religion and philosophy as branches of inquiry within which the NDE might be advantageously studied. The suggestion that NDEs actually are early stages in the movement of the life force into a noncorporeal state or "afterlife" has been an issue well discussed by major religions; and the possible existence of consciousness existing "outside" the human body is of intense interest to $\mathrm{mind} / \mathrm{brain}$ scientists and philosophers. However, Jansen also referred to "religious experiences that sometimes result" from psychedelic drug use. In describing his own ketamine experiences, he described "emergence into the light" (not $a$ light, but the light), and encountering " "God'" (his quotes).

This apparent ambivalence may reflect the general discomfort "mechanistic," "areligious," "objective" scientists feel when carefully examining subjective reports of NDEs, psychedelic drugs, and similar states. For while there are well-documented biological correlates and perhaps precursors of religious, NDE, and psychedelic states, the function, nature, and origin of these perceptions is not adequately addressed by a rigidly biological perspective. This is not because of any "antiscientific" axe to grind, but solely because those who describe these states, even those like Jansen who claim they are "nonreligious," routinely do so in religious and spiritual, rather than biological or adaptational, terms. And, the adaptive or biological significance of an endogenous NDE-ogen is difficult to discern.

Perhaps it is the nonmaterial nature of these phenomena that is so vexing to the materially-minded neuroscientist. For example, understanding how the television set works does not yield any information regarding from where the images and sounds arise. Nonmaterial subjective (and by its nature, wholly private) experience includes dreams, psychedelic and religious states, and NDEs. How do we approach the scientific study of phenomena that we cannot now measure with mechanical instruments, but can only verify with our subjective experience?

In these states, the body "drops away," as it were, but an ongoing, undeniable, compelling reality continues to be experienced. Brain metabolism continues, but what is being observed, and why is it no less "real" than objective, instrument-measurable, externally verifiable, consensually validated experience? Ketamine or "endopsychosin" effects on NMDA receptor function at discrete, strategic brain sites could be invoked, but this reductionism can become absurd. For ex- 
ample, is "everyday reality" as "real" and compelling as it is because of NMDA receptor function? Perhaps it is the case that the NMDA receptor is blockaded when the organism is perceiving a nonmaterial world!

Different versions of what constitutes "science" might lead to means of proceeding with the "scientific" study of "religious" phenomena. For example, Jansen stated that "within a scientific paradigm, it is not possible that the 'spirit rises out of the body . ..." Has this been disproved? Who has looked? And with what tools?

Jansen suggested that "the reproduction of NDEs by ketamine is unlikely to be a coincidence." This is because NMDA antagonists such as ketamine are neuroprotective, and have NDE-ogenic "side effects." From a "scientific" point of view, however, it is difficult to suggest that it is anything other than a coincidence. A coincidence is a correspondence of identity in space occupied or temporal position, or a remarkable occurrence of events at the same time or in the same way. If there is to be the maintenance of scientific credulity, the similarities of ketamine and other psychedelics to the NDE must be a coincidence. How could they be otherwise?

If the NDE-ogenic properties of endogenous neuroprotective agents are not a coincidence, the existence of these similarities would support one of two possible hypotheses. The first hypothesis would be that this association confers an evolutionary advantage on the organism or species. However, why would not a neuroprotective agent produce sensory and cognitive dulling, inhibition, and sedation, blurring the transition to unconsciousness and death? What is the adaptational or competitive advantage of an individuals' near-death state being a psychedelic or revelatory experience, of an otherworldly nature?

The second hypothesis is that the association supports a "nonverifiable" explanation (at least at this point in our scientific maturity); that is, that NDEs and ketamine/psychedelics establish the existence of nonmaterial realms.

Jansen rejected this latter hypothesis, and instead asserted that since ketamine-like compounds are released at death, these compounds (by no coincidence) produce NDE-like mental changes. There is, however, implicit in this, the assumption of a purposive (and not mechanical) cause directed to a definite end. That is, it is an explanation of nature in terms of utility or purpose. This is vitalism, or teleology, a distinctly religious or spiritual school of thought. 
The most cogent purposive reason for this association is that this indeed is an accurate preview of what happens at the time of death: a rapid transition to a nonmaterial realm, free-standing, autonomous, peopled by familiar and unfamiliar inhabitants. One may be forced to conclude that this is what does happen if it is not a coincidence. Freed of the somatic feedback, the conscious life force moves towards a nonmaterial realm, completely convinced of its veracity, and since there is no body, and therefore no ego (that is, no body-ego), this is the conscious moment inhabited by the mind.

If this were the case, then the release of ketamine-like or other endogenous psychedelics would be the brain's correlate of awareness leaving the body, rather than the cause of it "feeling that way." This would be similar to the fact that functional brain imaging techniques demonstrate identical findings regardless of whether perceptions are based upon external stimuli or upon imagined stimuli (Le Bihan, Turner, Zeffiro, Cuénod, Jezzard, and Bonnerot, 1993). They reflect correlates or mediators of perception, rather than attesting to the "reality" of what is perceived.

\section{References}

Abramson, H. A., Jarvik, M. E., Kaufman, M. R., Kornetsky, C., Levine, A., and Wagner, M. (1955). Lysergic acid diethylamide (LSD-25): I. Physiological and perceptual responses. Journal of Psychology, 39, 3-60.

Gillin, J. C., Kaplan, J., Stillman, R., and Wyatt, R. J. (1976). The psychedelic model of schizophrenia: The case of N,N-dimethyltryptamine. American Journal of Psychiatry, 133, 203-208.

Haertzen, C. A., and Hickey, J. E. (1987). Addiction Research Center Inventory (ARCI): Measurement of euphoria and other drug effects. In M. A. Bozarth (Ed.), Methods of assessing the reinforcing properties of abused drugs (pp. 489-524). New York, NY: Springer-Verlag.

Le Bihan, D., Turner, R., Zeffiro, T. A., Cuénod, C. A., Jezzard, P., and Bonnerot, V. (1993). Activation of human primary visual cortex during visual recall: A magnetic resonance imaging study. Proceedings of the National Academy of Sciences of the USA, 90, 11802-11805.

Linton, H. B., and Langs, R. J. (1962). Subjective reactions to lysergic acid diethylamide (LSD-25). Archives of General Psychiatry, 6, 352-368.

Olney, J. W., Labruyere, J., and Price, M. T. (1989). Pathological changes induced in cerebrocortical neurons by phencyclidine and related drugs. Science, 244, 1360-1362.

Olney, J. W., Labruyere, J., Wang, G., Wozniak, D. F., Price, M. T., and Sesma, M. A. (1991). NMDA antagonist neurotoxicity: mechanism and prevention. Science, 254, 1515-1518.

Pahnke, W. N., Kurland, A. A., Unger, S., Savage, C., and Grof, S. (1970). The experimental use of psychedelic (LSD) psychotherapy. Journal of the American Medical Association, 212, 1856-1863. 
Strassman, R. J. (1984). Adverse reactions to psychedelic drugs. A review of the literature. Journal of Nervous and Mental Disease, 172, 577-595.

Strassman, R. J. (1995). Hallucinogenic drugs in psychiatric research and treatment: Perspectives and prospects. Journal of Nervous and Mental Disease, 183, 127-138.

Strassman, R. J., Qualls, C. R., and Berg, L. M. (In press). Differential tolerance to biological and subjective effects of four closely-spaced doses of N,N-dimethyltryptamine in humans. Biological Psychiatry.

Strassman, R. J., Qualls, C. R., Uhlenhuth, E. H., and Kellner, R. (1994). Dose-response study of N,N-dimethyltryptamine in humans. II: Subjective effects and preliminary results of a new rating scale. Archives of General Psychiatry, 51, 98-108.

Szara, S. I. (1957). The comparison of the psychotic effects of tryptamine derivatives with the effects of mescaline and LSD-25 in self-experiments. In S. Garattini and V. Ghetti (Eds.), Psychotropic Drugs (pp. 460-467). New York, NY: Elsevier. 